\title{
FORENSIC ENGINEERING SURVEYS WITH UAV PHOTOGRAMMETRY AND LASER SCANNING TECHNIQUES
}

\author{
C. Cappelletti ${ }^{1}$, M. Boniardi ${ }^{1}$, A. Casaroli ${ }^{1}$, C. I. De Gaetani ${ }^{2 *}$, D. Passoni ${ }^{3}$, L. Pinto ${ }^{2}$ \\ ${ }^{1}$ Politecnico di Milano - DMEC (chiara.cappelletti@gmail.com, marco.boniardi@polimi.it, andrea.casaroli@polimi.it) \\ ${ }^{2}$ Politecnico di Milano - DICA (carloiapige.degaetani@polimi.it, livio.pinto@polimi.it) \\ ${ }^{3}$ Università di Genova - DICCA (daniele.passoni@ dicca.unige.it)
}

Commission II

KEY WORDS: UAV Photogrammetry, Terrestrial Laser Scanning, Forensic Engineering, Industrial Accidents Survey, 3D Imaging, Forensic Photography

\begin{abstract}
:
This work aims at presenting the use of new technologies in the field of forensic engineering. In particular, the use of UAV photogrammetry and laser scanning is compared with the traditional methods of surveying an accident site. In this framework, surveys must be carried out promptly, executed in a short time and performed so that the greatest possible amount of information is collected with sufficient accuracy to avoid the possibility of neglecting details once that the scene is no longer preserved. The combination of modern surveying techniques such UAV photogrammetry and laser scanning can properly fulfill these requirements. An experimental test has been arranged and instruments, procedures, settings, practical limits and results have been evaluated and compared with respect to the usual way of performing the survey for forensic purposes. In particular, both qualitative and quantitative considerations are given, assessing the completeness of the reconstructed model, the statistical evaluation of the errors and the accuracy achieved.
\end{abstract}

\section{INTRODUCTION}

One of the roles an engineer can cover in his professional life is that of an expert technician in a judicial proceeding. Whether the proceeding is a criminal or civil procedure, the judge and/or the parties involved may refer to an expert to determine, interpret, clarify and evaluate technical issues for which specific skills are required. In the technical-industrial field, the expert can be called, for example, to perform analysis of materials, components, machines and industrial plants, chemical analyses, safety assessments and appraisals, often in situations of civil, industrial or transport accidents. When doing so, an engineer is acting in the field of the forensic engineering, i.e. "the application of engineering principles to the investigation of failures or other performance problems", usually, within a legal context. In general, the purpose of forensic engineering is to determine causes of failure and it involves inspections, taking measurements, collecting evidences, performing experiments. The technical assessment often comes after the inspection of the accident site. Surveys must be carried out promptly, before the scene can be modified or deteriorated. It is necessary that they are executed in a short time, that the greatest possible amount of information is collected and with accuracy such that no detail can be neglected once that it can no longer be determined in the future. The traditional method of carrying out a survey of an accident site consists mainly in collecting photographic documentation and making measurements. Through the images it is possible to document the state of things, to visualize the area, to establish the position and shape of the parts and, by placing references on the side of the objects, their size. To be able to reconstruct position and dimensions of all objects it is necessary to take many measurements. In general, laser range meters and measuring tapes are used. Measurement operations can take a long time, especially over a very large area or with the presence of many parts and the possibility of introducing errors in

\footnotetext{
*Corresponding author
}

manual measurement operations due to human inaccuracy is very high. Moreover, it is not possible to measure every single detail of every single object. In order to overcome these problems and limitations, new technologies can be used to survey entire areas in every detail and in a short time, allowing to "crystallize" the accident scene in a three-dimensional model where all the necessary measurements can be taken; the adoption of these technologies has many advantages in terms of time, accuracy and results. Furthermore, these survey techniques consist in measuring objects without coming into direct contact with it, that is an aspect requiring not to be underestimated in some case. Range-based and image-based techniques, are well known in geomatics and could fullfill the requirements described above in the field of forensic engineering. In particular, a combination of terrestrial laser scans and photogrammetric drones surveys are suitable methodologies for such purpose. On the other hand, the instrumentation needed is often expensive, experience and training are crucial in both the surveying phase and the data processing. Comparing traditional methods with respect to such geomatic approach means taking into account all the aspects affecting the "economy" of a survey for forensic purposes, hence costs, efforts, duration of the operations, quality, accuracy. The problem of surveying traffic accidents has been approached in the frame of a specific and scientifically sound methodology at international level. The field of traffic accident reconstruction has been developed especially in the United States, where the associations involved lively debate on the subject. In 1985 the National Highway Traffic Safety Administration funded the preparation of national guidelines for the standardization of methodologies for the reconstruction of traffic accidents. Many authors have systematically addressed the task of outlining the correct surveying procedures (Fricke, 1990, Brach and Brach, 2011). From the technical point of view the procedure to be followed for a correct metric representation of the accident site does not present theoretical difficulties, but only operational ones, as a consequence of the wide variety of en- 
vironmental difficulties and cases. The use of photogrammetry has long been included among the surveying methods (Du et al., 2009, Buck et al., 2007, Randles et al., 2010, Fraser et al., 2005, Carter et al., 2016). Recently, also laser scans are used for surveying accidents (Pagounis et al., 2006, Buck et al., 2007, Pu et al., 2011, Eyre et al., 2017, Fowle and Schofield, 2011). In this context, unconventional methods of low-cost surveying have also been tested, for example with particular devices such as Microsoft Kinect (Colwill, 2016). However, following the latest developments of geomatics of the last years it is important to define in a clear and exportable way a methodology for surveying in such kind of subject with known precision and reliability. In this sense, the advent of the Unmanned Aerial Vehicle (UAV) opens new scenarios thanks to speed, completeness and precision that the instrument offers as described in (Smith, 2015, Ardestani et al., 2016, Su et al., 2016).

\section{EXPERIMENTAL TEST}

A staged car accident has been considered as test case to be surveyed simulating a simple example of forensic engineering. The simulated accident site have been surveyed with both the traditional approach and the geomatics approach by integrating laser scans and UAV photogrammetry. The survey was carried out in Sarmato (Piacenza, Italy) in December 2017. As a place of the accident, a short tunnel passing underneath Sarmato's town hall building has been chosen. The tunnel had an irregular shape and size L x W x H of about $11.5 \mathrm{~m} \times 4.9 \mathrm{~m} \times 4.4 \mathrm{~m}$. The choice of a tunnel has been made with the purpose of highlighting pros and cons of the tested technologies and the usefulness of their combination. Even if a staged car accident is a small-scale simulation, this situation has many common features with a real case, e.g., the presence of different objects with different size arranged in a disorderly way, the presence of different materials such as metal, glass, cladding materials and construction materials, the presence of parts separated by vehicles, the fact that part of the accident is located within a semi-enclosed space and part outdoors. All these elements make this scenario compatible with a real outdoor accident (for example, a train accident) or a real industrial accident inside a building (for example, the explosion of a machine). Furthermore, the presence of materials such as glass and metallic surfaces allows to study the response of the instruments on particularly problematic surfaces. In the simulated accident site three medium size cars (named C1, C2, C3) and two car wheels (named W1 and W2) were present. The cars were positioned inside the tunnel in order to occupy the entire length and so that at least one of these protruded outside. Figure 1 shows a sketch of the simulated accident site.

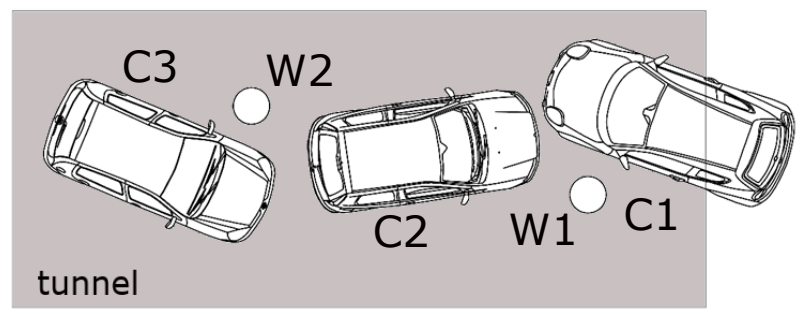

Figure 1. Simulated accident site

\subsection{GNSS ground points survey}

Four points have been defined as station points for laser scanning the accident site, named points $100,200,300,400$. Stations 100 and 200 were located outside at the opposite sides of the tunnel and signalized with black/white high-contrast signals while stations 300 and 400 were inside, signalized by signs drawn with a chalk and not too close to the objects to be scanned (at about $2 \mathrm{~m}$ of distance). Furthermore, seventeen additional sparse points have been chosen to support UAV survey as Ground Control Points (GCPs) and Check Points (CPs). These points were signalized by three different types of targets: black/yellow highcontrast signals, road cones, manholes covers. The use of targets with so different characteristics have been considered as part of the analysis. In total, six high-contrast signals, six manholes covers and five road cones have been positioned in the area surrounding the tunnel. The coordinates have been acquired with a GNSS Leica Viva GS14 receiver in Real Time Kinematic (RTK) mode. The declared accuracy of the receiver in such mode is $8 \mathrm{~mm}$ in horizontal direction and $15 \mathrm{~mm}$ in vertical direction. In particular, the coordinates of the point at the center of the high-contrast signals and of the two opposite vertices of the manholes covers and road cones have been acquired, for a total of thirty-two points. Their distribution is shown in Figure 2. The coordinates of the center of the manholes covers and road cones have been calculated later using their acquired vertices while the coordinates of stations 300 and 400, being them in an indoor environment, have been measured with topographic methods using stations 100 and 200 as references.

\subsection{Tunnel laser scanning}

With stations 100, 200, 300 and 400 georeferenced, four laser scans have been performed using a Leica Nova MultiStation MS60 (MS) (Fagandini et al., 2017) from each of them. The scans have been set on the basis of different aspects, for instance the size of the smallest detail of the surface that must be recognized and taking into account that the higher the resolution is and the more time-consuming the scan is (also considering that with a resolution better than the accuracy of the instrument the scan would be oversampled and a more noisy and less accurate result would be obtained). Based on these considerations, the first scan has been performed from station 100 with a resolution of $20 \mathrm{~mm}$ x $20 \mathrm{~mm}$ at about $18 \mathrm{~m}$ of distance obtaining about 95000 back scatterers. The second scan from station 200 had a resolution of $20 \mathrm{~mm} \mathrm{x}$ $20 \mathrm{~mm}$ at a distance of about $12 \mathrm{~m}$ obtaining a cloud of about 87000 points. Regarding the scans from stations 300 and 400 the resolution set has been $20 \mathrm{~mm} \times 20 \mathrm{~mm}$ at $2 \mathrm{~m}$ of distance for both obtaining about 67000 and 34000 back scatterers, respectively.

\subsection{UAV photogrammetric survey of the area}

The photogrammetric survey has been carried out using a Parrot Bebop 2 (Pagliari and Pinto, 2018), a small quadcopter drone falling into the category of ultra-light and low-cost UAVs, equipped with a fisheye 14MP camera and controlled remotely by the Pix4Dcapture mobile application. Once the flight has been set, it is performed automatically following the flight plan. A total of three flights have been performed, with a duration of approximately 56 minutes each, at a height of $30 \mathrm{~m}$ above the ground and over a square area of approximately $50 \mathrm{~m}$ per sides. The height has been determined in such a way as to have enough margin on the roofs and avoid the taller buildings. Flights had different flight plans in order to study the differences in terms of results and being able to determine which was the best solution. In particular the first 


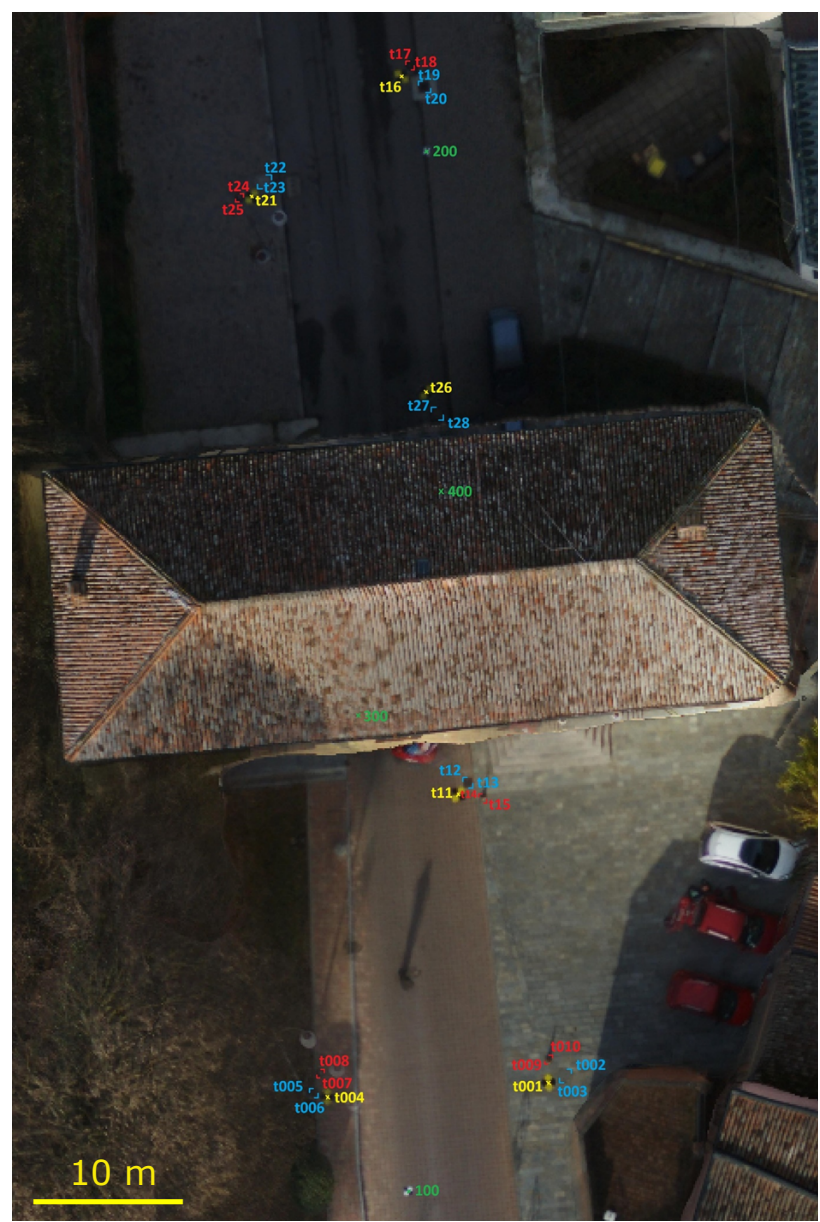

Figure 2. Points acquired. Station points (green), high-contrast signals (yellow), road cones (red), manholes covers (blue)

flight has been made with a double grid plan and an almost square geometry, with the diagonal oriented as the tunnel axis (named square flight), the second flight was made with a double grid plan and a rectangular geometry, with the same orientation of the tunnel (named rectangular flight) and the third flight was made with a circular plan, with the camera tilted towards the center where the tunnel is located (named circular flight). Figure 3 gives an overview of the different flight plans.
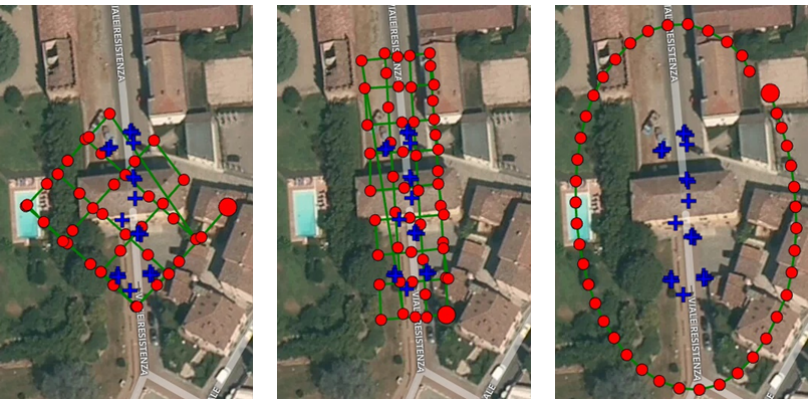

Figure 3. Flight plans. Square (left), Rectangular (centre), Circular (right). Grid points (red), ground points (blue)

\subsection{Manual measurements}

The last phase of the survey consisted in making manual measurements for comparison. A manual survey is usually done by taking manual measurements of the accident scene, the area in which it is inserted and the position of the objects, to be combined with photographic documentation. During the survey a Canon EOS 5D Mark III camera equipped with two lenses (wide angle 16$35 \mathrm{~mm}$, telephoto 70-200mm), a Leica Disto D2 laser range meter and tape measures have been used. Photos of the whole area have been taken, with both general and particular views. The tunnel and the objects have been measured manually and removed once that their position was marked on the asphalt with a chalk. Using these reference marks, all the necessary measurements have been taken. The positions of the wheels have been signalled by means of four curves along the outer circumference, while those of the car by means of the tire size and the position of the wheel hub. The measurements have been made with respect to the tunnel extents.

\subsection{Data processing}

GPS and laser scan acquisitions have been processed with the software Leica Infinity. As output, the WGS84-UTM coordinates of the 32 measured points and 4 georeferenced point clouds have been obtained. Once that the center of the manholes covers and road cones have been computed on the basis of the coordinates of their vertices, the coordinates of the nineteen points outside the tunnel have been used for processing the photogrammetric survey. Drone's images have been processed with the photogrammetric software Pix4Dmapper 4.2.25. First, matches between the frames have been found and used to re-align them. From these initial matches, an Automatic Aerial Triangulation (AAT) and a Bundle Block Adjustment (BBA) have been run to determine the external orientation of the frames. Tie Points (TPs) for images block calibration have been automatically detected by the software on overlapped images while GCPs for model georeferencing and CPs have been collimated manually. Based on the georeferenced model and the TPs previously created, the point cloud and the 3D mesh have been generated. The point cloud has been densified by the software with a procedure called Cloud Point Densification where additional TPs are created, obtaining a dense point cloud. On such dense point cloud a 3D textured mesh and orthomosaics have been generated. The workflow just described above is sketched in Figure 4. The whole procedure has been replicated for the three different flight plans and by varying the set of GCPs and CPs (among points signalized with high-contrast signals, manholes covers or road cones).

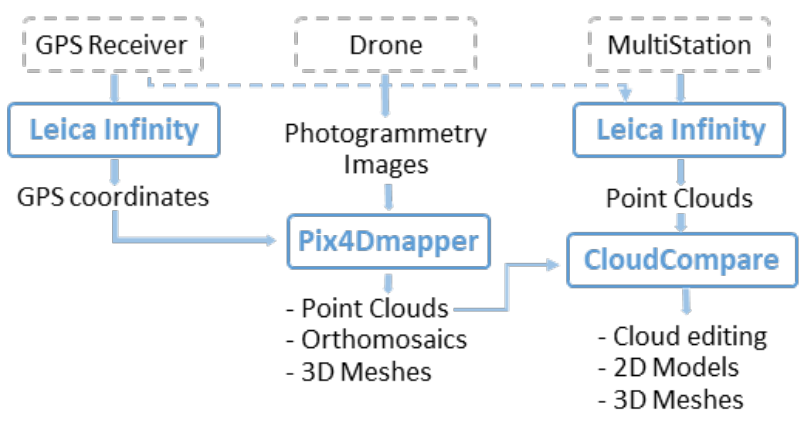

Figure 4. Processing workflow

\section{RESULTS}

Point clouds exported by Leica Infinity and Pix4Dmapper have been managed by the open source software CloudCompare 2.10. Through CloudCompare point clouds obtained by photogrammetry and laser scans have been cleaned, compared, measured, merged and used to extract 2D models and 3D meshes. Since 
both point clouds produced by Pix4Dmapper and those extracted from MS are georeferenced, they were automatically aligned and did not need to be registered between them.

\subsection{UAV photogrammetry results}

Several Pix4Dmapper processings have been assessed, evaluating the quality of the output on the basis of aspects such as the completeness of the point clouds and 3D meshes, voids, distortions or macroscopic reconstruction errors, the GCPs and CPs errors (absolute values, Root Mean Square (RMS), projection errors). Firstly, the three different types of flight plan have been compared. For each of them the same five black/yellow high contrast signalized points have been used as GCPs. The sixth one have been excluded because resulted in shadow and difficult to be matched by the software, consequently. The code of these points were $\mathrm{t} 001, \mathrm{t} 004, \mathrm{t} 11, \mathrm{t} 16, \mathrm{t} 21$. The three flight plans contemplated square, rectangular and circular flights at the same flight height. The square flight has been performed with a double grid plan and an almost square geometry, with the diagonal oriented as the tunnel axis. 39 images have been acquired and processed obtaining an average Ground Sample Distance (GSD) of $6.90 \mathrm{~cm}$ and a dense point cloud with average density of 14.50 points per $\mathrm{m}^{3}$. The model reconstructs well the horizontal surfaces parallel to the ground, while the vertical surfaces present some problems: the point cloud is incomplete and the mesh partially deformed. It is possible to see both the entrances of the tunnel and through them part of the cars of the accident scene but the reconstruction is distorted (see Figure 5).
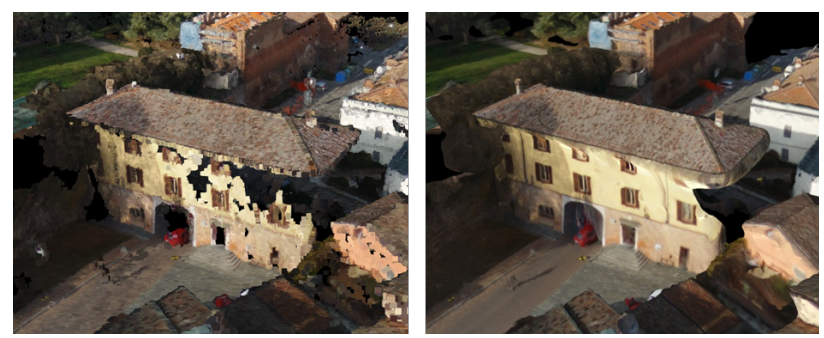

Figure 5. Square flight, point cloud (left) and mesh (right)

RMS of the GCPs errors is equal to $0.011 \mathrm{~m}$ while the projection error have RMS of 1.261 pixels. The rectangular flight has been performed with a double grid plan and rectangular geometry. 53 images were acquired obtaining an average Ground Sample Distance (GSD) of $6.17 \mathrm{~cm}$ and a dense point cloud with average density of 18.18 points per $\mathrm{m}^{3}$. In this case the point cloud on the front facades of the building is quite complete and the reconstruction is better and less distorted than in the case of square flight. Through the tunnel entrances it is possible to clearly see part of the cars of the accident scene. The side facades of the building have not been acquired due to the flight plan geometry (see Figure 6). RMS of the GCPs errors and projection error are $0.008 \mathrm{~m}$ and 1.347 pixels, respectively. The circular flight has been performed with a circular plan, with the camera tilted towards the center of the flight area where the town hall building is located. 44 images have been acquired obtaining an average GSD of $4.20 \mathrm{~cm}$ and a dense point cloud with average density of 42.01 points per $\mathrm{m}^{3}$. With this flight plan the building has been completely reconstructed, without lacks in the point cloud and without evident distortions in the mesh. Part of the accident is visible through the tunnel openings and the cars have been well reconstructed (see Figure 7). GCPs errors have RMS equal to $1.2 \mathrm{~cm}$ while the projection error have RMS of 0.495 pixels.
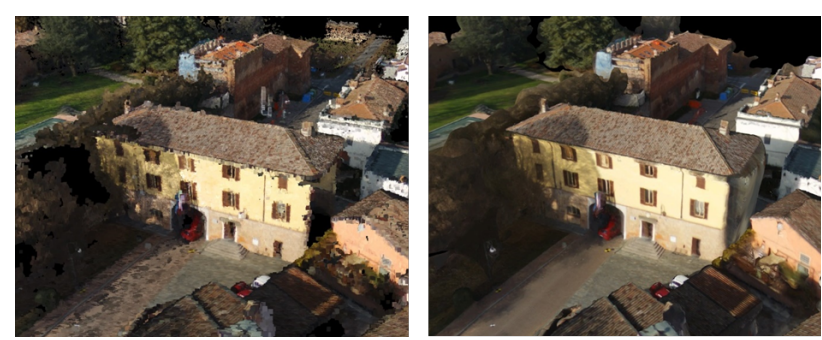

Figure 6. Rectangular flight, point cloud (left) and mesh (right)
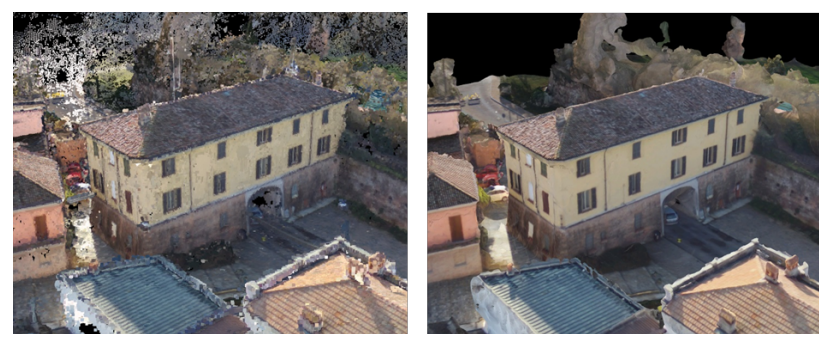

Figure 7. Circular flight, point cloud (left) and mesh (right)

GCPs error RMS obtained by just the rectangular and circular flight plans are almost equal while the projection errors shows to be different, as shown in Table1.

\begin{tabular}{|l|c|c|c|c|}
\hline GCP & $\mathrm{e}_{\mathrm{X}}[\mathrm{cm}]$ & $\mathrm{e}_{\mathrm{Y}}[\mathrm{cm}]$ & $\mathrm{e}_{\mathrm{Z}}[\mathrm{cm}]$ & $\mathrm{e}_{\text {projection }}[\mathrm{pixel}]$ \\
\hline \hline $\mathrm{t} 001$ & -1.0 & -0.4 & 0.4 & 1.350 \\
$\mathrm{t} 004$ & 0.3 & -0.7 & 0.2 & 1.575 \\
$\mathrm{t} 11$ & 1.1 & 1.7 & -2.0 & 1.046 \\
$\mathrm{t} 16$ & -0.2 & -0.7 & 0.2 & 1.319 \\
$\mathrm{t} 21$ & -0.1 & 0.5 & 0.0 & 1.392 \\
\hline RMS & 0.7 & 0.9 & 0.9 & 1.347 \\
\hline \hline $\mathrm{t} 001$ & -0.6 & -0.7 & 1.0 & 0.522 \\
$\mathrm{t} 004$ & -0.8 & 0.3 & -0.2 & 0.507 \\
$\mathrm{t} 11$ & 2.3 & 0.7 & -1.8 & 0.442 \\
$\mathrm{t} 16$ & -1.3 & -1.8 & 0.2 & 0.519 \\
$\mathrm{t} 21$ & 0.5 & 2.6 & 1.0 & 0.480 \\
\hline RMS & 1.3 & 1.5 & 1.0 & 0.495 \\
\hline
\end{tabular}

Table 1. GCPs errors summary: Rectangular flight (Top), Circular flight (bottom)

High projection errors are typically due to inaccurately marked points on images or incorrectly aligned images and should be less than 1 pixel. The circular flight plan has the lowest value due to the best alignment between the images thanks to the higher number of matched points per image. Furthermore, it obtains the more complete and undistorted reconstruction. On the other hands, the generated orthomosaic is the one with the most missing or distorted areas. The orthomosaic can present distortions because it is generated by orthorectification that removes the perspective distortions from images using the DSM generated from the densified point cloud so that the distances are preserved and the orthomosaic can be used to make measurement. In any case, it is possible to edit the orthomosaic to eliminate the distortions present. After comparing the three different flight plans, the result is that the circular one is the more appropriate for the purposes of the experiment. Having established the reference flight plan it has been investigated the difference in using different types of ground targets. The three types of target used during the survey have been high-contrast signals (named type A) designed for pho- 
togrammetric purposes, manholes covers (type B) and road cones (type C). Using alternatively a type as GCPs and the others as CPs the evaluation has been been made on GCPs errors, CPs errors and their RMS. Two points resulted in shadow and have been excluded from the analysis. The high contrast signalized points were named $\mathrm{t} 001, \mathrm{t} 004, \mathrm{t} 11, \mathrm{t} 16$ and $\mathrm{t} 21$, the road cones were centred on points $\mathrm{t} 910, \mathrm{t} 78, \mathrm{t} 1415, \mathrm{t} 1718$ and $\mathrm{t} 2425$ while the centre of the manhole covers were points $\mathrm{t} 23, \mathrm{t} 56, \mathrm{t} 1213, \mathrm{t} 1920$ and t2223. Firstly, the five black/yellow high contrast signals have been used as GCPs while the remaining ten as CPs. In terms of GCPs error, results are practically the same as done previously when assessing the circular flight plan. Regarding the CPs errors, almost all of them show errors lower than $2^{*} \mathrm{GSD}$ (GSD obtained was $4.19 \mathrm{~cm}$ ) in horizontal directions and lower than $3 * \mathrm{GSD}$ in vertical direction, that are the threshold values of the accuracy expected for a well reconstructed model. The errors in $\mathrm{Z}$ direction are particularly high for road cones. This was expected, because the $\mathrm{Z}$ coordinate of these CPs has been obtained by adding the height of the cones to the vertical GPS coordinate acquired at ground level, thus introducing a source of inaccuracy. The RMS of the error on CPs is $2.1 \mathrm{~cm}, 2.7 \mathrm{~cm}$ and $7.3 \mathrm{~cm}$ in $\mathrm{X}, \mathrm{Y}$ and $\mathrm{Z}$ directions, respectively. Results are summarized in Table2.

\begin{tabular}{|l|c|c|c|c|c|}
\hline GCP & type & $\mathrm{e}_{\mathrm{X}}[\mathrm{cm}]$ & $\mathrm{e}_{\mathrm{Y}}[\mathrm{cm}]$ & $\mathrm{e}_{\mathrm{Z}}[\mathrm{cm}]$ & $\mathrm{e}_{\mathrm{proj}}[\mathrm{px}]$ \\
\hline t001 & $\mathrm{A}$ & -0.6 & -0.8 & 1.3 & 0.516 \\
t004 & $\mathrm{A}$ & -1.0 & 0.4 & -1.1 & 0.494 \\
t11 & $\mathrm{A}$ & 2.2 & 0.8 & -1.4 & 0.440 \\
t16 & $\mathrm{A}$ & -1.1 & -1.7 & -0.6 & 0.535 \\
t21 & $\mathrm{A}$ & 0.5 & 2.6 & 1.9 & 0.490 \\
\hline RMS & & 1.2 & 1.5 & 1.3 & \\
\hline \hline CP & type & $\mathrm{e}_{\mathrm{X}}[\mathrm{cm}]$ & $\mathrm{e}_{\mathrm{Y}}[\mathrm{cm}]$ & $\mathrm{e}_{\mathrm{Z}}[\mathrm{cm}]$ & $\mathrm{e}_{\text {proj }}[\mathrm{px}]$ \\
\hline t23 & $\mathrm{B}$ & -0.4 & -4.0 & -3.3 & 0.511 \\
t56 & $\mathrm{B}$ & 4.6 & 6.1 & 0.1 & 1.385 \\
t1213 & $\mathrm{B}$ & 1.3 & 2.5 & -6.2 & 0.265 \\
t1920 & $\mathrm{B}$ & 1.2 & -1.3 & -3.0 & 0.277 \\
t2223 & $\mathrm{B}$ & 0.2 & 2.8 & 0.9 & 0.743 \\
t910 & $\mathrm{C}$ & 2.8 & -2.1 & 11.5 & 0.674 \\
t78 & $\mathrm{C}$ & 3.3 & -2.3 & 14.8 & 0.810 \\
t1415 & $\mathrm{C}$ & 0.3 & 2.0 & 10.5 & 0.415 \\
t1718 & $\mathrm{C}$ & 1.4 & -0.7 & 4.0 & 0.615 \\
t2425 & $\mathrm{C}$ & 2.0 & 0.2 & 9.8 & 1.088 \\
\hline RMS & & 2.1 & 2.7 & 7.3 & \\
\hline
\end{tabular}

Table 2. High-contrast target as GCPs, errors on GCPs and CPs

Road cones have been then used as GCPs. The road cones have been chosen to investigate the possibility of using movable elements typically already present on the scene of an accident, thus eliminating the need to place specific signals. In this case, the mean RMS of the GCPs errors is $0.011 \mathrm{~m}$. Regarding the CPs, most of the errors in the $\mathrm{X}$ and $\mathrm{Y}$ directions are lower than the GSD value $(4.07 \mathrm{~cm})$ while almost all the errors in the $\mathrm{Z}$ direction are greater than the value of GSD, but still lower or comparable with the $3 *$ GSD limit. This may be due to the type of GCPs used, as explained in the previous case. Since the cones have been used as GCPs, the software used these points to reconstruct the model trying to match the corresponding reconstructed $3 \mathrm{D}$ points to the input GPS coordinates: for this reason, the whole model is likely to have an error in the $\mathrm{Z}$ direction. This error is revealed by errors on the CPs, which in fact show a difference along $\mathrm{Z}$ between the reconstructed position and the acquired GPS coordinates. The RMS of the error on CPs is $2.1 \mathrm{~cm}, 3.4 \mathrm{~cm}$ and $11.4 \mathrm{~cm}$ in $\mathrm{X}, \mathrm{Y}$ and $\mathrm{Z}$ directions, respectively. Table 3 summarizes the results.

Finally, the manholes covers have been used as GCPs. Such kind of target has been chosen to study the possibility of using fixed el-

\begin{tabular}{|l|c|c|c|c|c|}
\hline GCP & type & $\mathrm{e}_{\mathrm{X}}[\mathrm{cm}]$ & $\mathrm{e}_{\mathrm{Y}}[\mathrm{cm}]$ & $\mathrm{e}_{\mathrm{Z}}[\mathrm{cm}]$ & $\mathrm{e}_{\text {proj }}[\mathrm{px}]$ \\
\hline $\mathrm{t} 910$ & $\mathrm{C}$ & 0.0 & -0.9 & 0.0 & 0.690 \\
t78 & $\mathrm{C}$ & 1.0 & -0.5 & -0.5 & 0.850 \\
$\mathrm{t} 1415$ & $\mathrm{C}$ & -1.6 & 2.8 & 0.1 & 0.427 \\
$\mathrm{t} 1718$ & $\mathrm{C}$ & 1.6 & -0.9 & -1.1 & 0.622 \\
t2425 & $\mathrm{C}$ & -1.6 & 0.6 & 0.9 & 1.051 \\
\hline RMS & & 1.3 & 1.4 & 0.6 & \\
\hline \hline $\mathrm{CP}$ & type & $\mathrm{e}_{\mathrm{X}}[\mathrm{cm}]$ & $\mathrm{e}_{\mathrm{Y}}[\mathrm{cm}]$ & $\mathrm{e}_{\mathrm{Z}}[\mathrm{cm}]$ & $\mathrm{e}_{\text {proj }}[\mathrm{px}]$ \\
\hline t23 & $\mathrm{B}$ & -3.0 & -2.6 & -14.6 & 0.521 \\
t56 & $\mathrm{B}$ & 2.4 & 8.5 & -15.6 & 1.384 \\
t1213 & $\mathrm{B}$ & -0.2 & 3.3 & -16.6 & 0.274 \\
t1920 & $\mathrm{B}$ & 1.5 & -1.4 & -8.0 & 0.279 \\
t2223 & $\mathrm{B}$ & 0.7 & 3.3 & 7.0 & 0.770 \\
t001 & $\mathrm{A}$ & -3.0 & 0.5 & -10.3 & 0.521 \\
t004 & $\mathrm{A}$ & -3.0 & 2.2 & -13.2 & 0.499 \\
t11 & $\mathrm{A}$ & 0.7 & 1.7 & -12.0 & 0.442 \\
t16 & $\mathrm{A}$ & -0.7 & -1.8 & -5.9 & 0.525 \\
t21 & $\mathrm{A}$ & 0.9 & 3.1 & -6.8 & 0.492 \\
\hline RMS & & 2.1 & 2.7 & 11.4 & \\
\hline
\end{tabular}

Table 3. Road cones as GCPs, errors on GCPs and CPs

ements belonging to the area to be surveyed. This possibility has several advantages, as it is not necessary to place any signal and it is not even necessary to access the area itself (for example in the case of a dangerous area). In fact, as fixed elements, it is possible to acquire their GPS coordinates at a later time, even days after surveying, for example once the area of the accident has already been cleared. With the flight already performed, it is possible to decide which elements are better to be used as targets, looking for on the images the most visible elements. Furthermore, it is also possible to use them several times to perform more surveys with the same GCPs. In this test, the GCPs errors are higher than the previous one, with a mean RMS of $0.021 \mathrm{~m}$. Furthermore, three CPs show particularly high errors in $\mathrm{Z}$ direction. All these points are road cones, so again the reason could be related to the accuracy with which the tip of the cones have been measured. The RMS of the CPs errors is $1.7 \mathrm{~cm}, 2.3 \mathrm{~cm}$ and $8.7 \mathrm{~cm}$ for $\mathrm{X}, \mathrm{Y}$ and $\mathrm{Z}$ direction, respectively, as shown in Table4.

\begin{tabular}{|l|c|c|c|c|c|}
\hline GCP & type & $\mathrm{e}_{\mathrm{X}}[\mathrm{cm}]$ & $\mathrm{e}_{Y}[\mathrm{~cm}]$ & $\mathrm{e}_{\mathrm{Z}}[\mathrm{cm}]$ & $\mathrm{e}_{\text {proj }}[\mathrm{px}]$ \\
\hline $\mathrm{t} 23$ & $\mathrm{~B}$ & -1.2 & -5.0 & 1.9 & 0.337 \\
$\mathrm{t} 56$ & $\mathrm{~B}$ & 2.0 & 4.1 & 2.3 & 1.014 \\
$\mathrm{t} 1213$ & $\mathrm{~B}$ & 0.2 & 0.9 & -3.7 & 0.263 \\
$\mathrm{t} 1920$ & $\mathrm{~B}$ & -1.2 & -0.6 & 1.8 & 0.280 \\
t2223 & $\mathrm{B}$ & -0.2 & 0.9 & -1.2 & 0.769 \\
\hline RMS & & 1.2 & 2.9 & 2.3 & \\
\hline \hline $\mathrm{CP}$ & type & $\mathrm{e}_{\mathrm{X}}[\mathrm{cm}]$ & $\mathrm{e}_{\mathrm{Y}}[\mathrm{cm}]$ & $\mathrm{e}_{\mathrm{Z}}[\mathrm{cm}]$ & $\mathrm{e}_{\text {proj }}[\mathrm{px}]$ \\
\hline $\mathrm{t} 910$ & $\mathrm{C}$ & 1.3 & -3.5 & 16.0 & 0.672 \\
t78 & $\mathrm{C}$ & 1.9 & -3.5 & 17.9 & 0.845 \\
$\mathrm{t} 1415$ & $\mathrm{C}$ & -1.0 & 0.5 & 13.1 & 0.433 \\
$\mathrm{t} 1718$ & $\mathrm{C}$ & 1.0 & -2.7 & 2.2 & 0.616 \\
$\mathrm{t} 2425$ & $\mathrm{C}$ & -2.3 & -1.6 & 7.6 & 1.060 \\
$\mathrm{t} 001$ & $\mathrm{~A}$ & -2.0 & 2.2 & 6.0 & 0.514 \\
$\mathrm{t} 004$ & $\mathrm{~A}$ & -2.3 & -0.9 & 2.1 & 0.491 \\
$\mathrm{t} 11$ & $\mathrm{~A}$ & 1.1 & -0.8 & 1.2 & 0.439 \\
$\mathrm{t} 16$ & $\mathrm{~A}$ & 1.5 & -3.8 & -2.4 & 0.530 \\
$\mathrm{t} 21$ & $\mathrm{~A}$ & 0.8 & 0.7 & -0.2 & 0.491 \\
\hline RMS & & 1.7 & 2.3 & 8.7 & \\
\hline
\end{tabular}

Table 4. Manhole covers as GCPs, errors on GCPs and CPs 


\subsection{Laser scans results}

In total, four laser scans have been made from four different stations, two of which from the outside (stations on points 100 and 200, named S100 and S200) and two from inside the tunnel (on points 300 and 400, named S300 and S400). The resulting point clouds were already georeferenced and oriented in space but there were no points of known coordinates to be used as check points to evaluate the absolute accuracy. For this reason, it is assumed that the accuracy is equal to that declared by the manufacturer $(2 \mathrm{~mm}$ $+2 \mathrm{ppm}$ ), although this accuracy may be subjected to variations due to several factors, e.g. distance of the reflectors and angle of incidence. S100 has acquired 95030 points in a volume of about $62472 \mathrm{~m}^{3}$. This scan has almost entirely acquired the back of C1 and partially the front of $\mathrm{C} 3$ and $\mathrm{W} 1$, while the position of $\mathrm{C} 2$ can only be distinguished by the acquisition of some parts of it. The bodywork of $\mathrm{C} 2$ has not been acquired due to the reflectivity characteristics of its surface which is a dark metallic paint. In fact, very dark surfaces are difficult to be acquired due to the high degree of absorbed energy while metallic surfaces due to the too high reflectivity. The details of the car such as wheels, lights, windows and windshield frames, interior details have been correctly acquired because made of different materials. The surfaces of the windows have not been acquired as the laser passed through the glass and in some cases the interior surfaces have been acquired instead. Regarding the other scan carried out from outside the tunnel S200, 87554 points have been acquired in a volume of about $52412 \mathrm{~m}^{3}$. In this case the acquisition comprised mainly the back of $\mathrm{C} 3$, part of the front of $\mathrm{C} 1, \mathrm{~W} 2$ and some details of C2. S300 and S400 have been carried out inside the tunnel to be able to better acquire the sides of the cars and the parts not visible from the outside. In these cases, the scans have been performed at very close distances from the target objects. S300 acquired 67165 points in a volume of about $2220 \mathrm{~m}^{3}$ but $\mathrm{C} 2$ still presented a poor acquisition despite the close distance and the best angle of incidence. S400 aimed at better acquiring the sides of $\mathrm{C} 2, \mathrm{C} 3$ and W2. It has acquired 34697 points in a volume of about $1540 \mathrm{~m}^{3}$

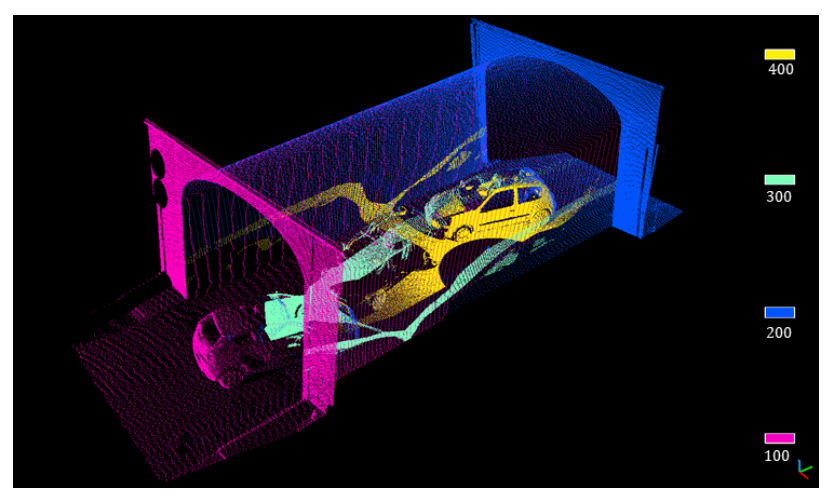

Figure 8. Point cloud after combining the four scans.

and while the bodywork of $\mathrm{C} 3$ has been correctly acquired, the bodywork of $\mathrm{C} 2$ and $\mathrm{W} 2$ have not been acquired at all, probably because too close to the instrument. From the combination of the four scans it is possible to obtain an almost complete reconstruction of the objects (apart from C2).The complete point cloud is shown in Figure 8. The positioning of the stations proved to be particularly complicated, since the tunnel was very narrow and the space available inside was little, otherwise it would have been possible to carry out the scans so as not to have shielded parts. The bodywork of $\mathrm{C} 2$ has not been acquired by any of the scans due to the problems of reflectivity but thanks to the many details that have been acquired (including lights, wheels, window frames), it is easy to identify its shape, dimensions and position. Therefore it is possible to take the measurements anyway and reconstruct the object.

\subsection{UAV photogrammetry-laser scans integration}

Point clouds obtained by photogrammetry and laser scans have been integrated to obtain a better, more complete and more accurate reconstruction of the accident site. In particular the dense point clouds obtained with the circular flight plan using the high contrast signals as GCPs and the combined laser scans have been merged (see Figure 9).

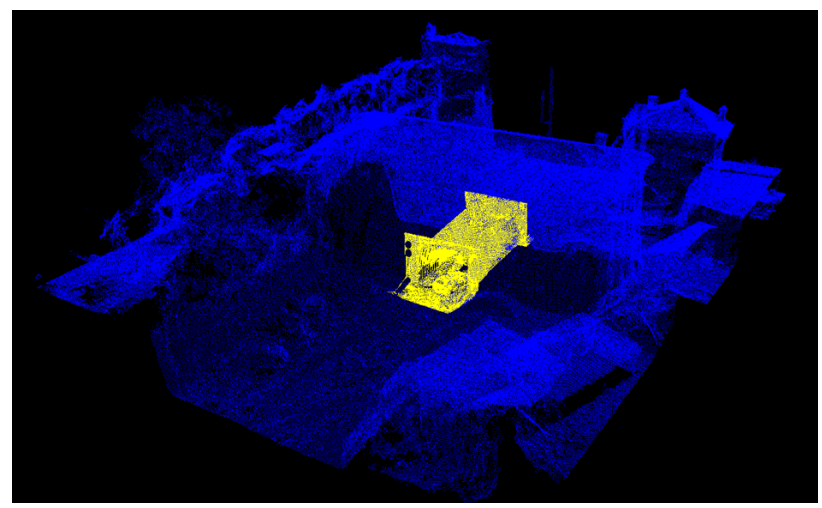

Figure 9. Final point cloud merging the point clouds obtaned by laser scans (yellow) and UAV photogrammetry (blue).

Points inside the tunnel acquired by photogrammetry have been deleted. The point cloud resulting from the integration of the two techniques is completely coloured (RGB colors), georeferenced and oriented in space; each point is characterized by a set of relative coordinates, a set of absolute coordinates (E, N, height) and a set of RGB values, and, only for the points scanned by laser, also by a scalar value indicating the intensity of the beam returned to the instrument. The part generated by photogrammetry has an estimated absolute accuracy (that is, with respect to absolute geographic coordinates) of $4.23 \mathrm{~cm}$ in the horizontal direction and $2.72 \mathrm{~cm}$ in the vertical direction and a relative accuracy of $1.7 \mathrm{~cm}$. The part generated by laser scanning has an assumed accuracy of $2 \mathrm{~mm}$, which is the one declared by the manufacturer. The point cloud can be used to make measurements, to determine the absolute and relative positions of each point, to compute 3D models and extract 2D models (sections). The final 3D mesh has been obtained by merging the mesh computed by Pix4dmapper (for the part coming from UAV photogrammetry, see Figure 10) and the mesh computed with CloudCompare. The construction of the mesh inside the tunnel has been done with CloudCompare and it has proved to be particularly problematic due to the empty areas in correspondence of windows, shielded parts and the bodywork of $\mathrm{C} 2$. 2D sections of the point cloud inside the tunnel have been obtained by slicing the model in different directions and then projecting the slices onto a 2D plane (Figure 11). By importing them into a CAD software contour lines have been drawn (e.g. to create the planimetries of the area) to make measurements.

\subsection{Comparison with manual measurements and discussion}

During the survey, several measurements have been made by hand to be able to make a comparison between the traditional (manual) method and the model reconstructed by means of photogrammetry and laser scanning. The measurements have been taken with a laser distance meter and two tape measures ( $3 \mathrm{~m}$ and $10 \mathrm{~m}$ long) 


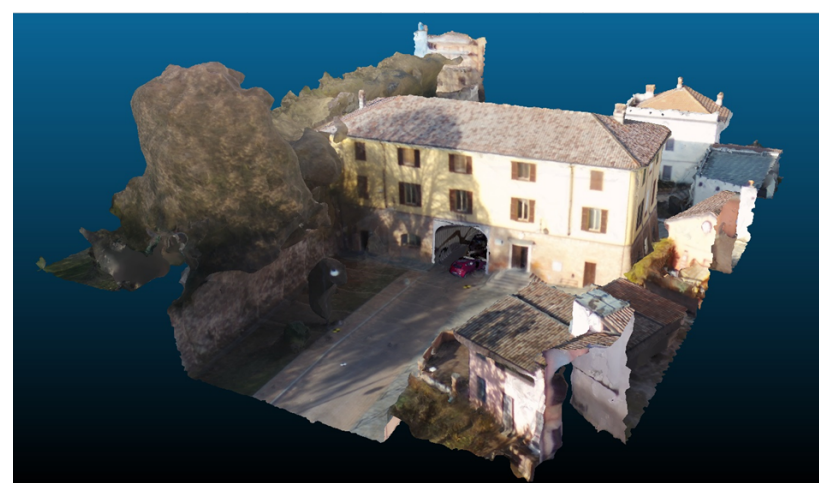

Figure 10. Final 3D mesh.
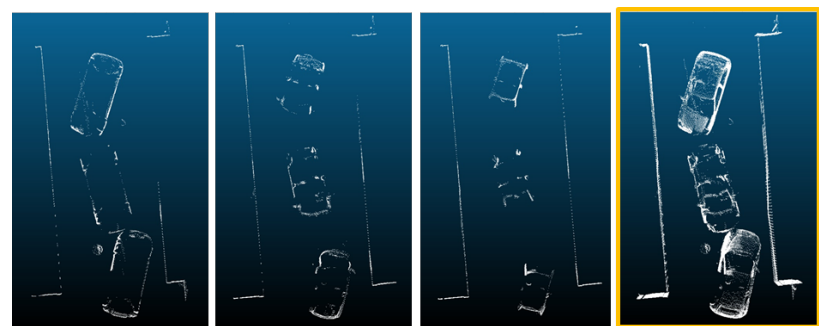

Figure 11. Sections of the point cloud inside the tunnel. On the right the $2 \mathrm{D}$ model obtained by overlapping several of them.

with a declared accuracy of $1.5 \mathrm{~mm}, 0.9 \mathrm{~mm}$ and $2.3 \mathrm{~mm}$, respectively. Buildings, surrounding area and details of the accident scene have been measured in terms of object dimensions and object positions. Manual measurements of objects dimensions (such as windows, doors, steps, car details, building details) can be considered fairly accurate while measurements of the positions of the objects inside the tunnel proved to be particularly problematic. The measurement of the positions of objects on the scene of an accident is in fact generally difficult to perform with accuracy due to the lack of certain references: in this case they have been measured using the tunnel walls or ends as a reference, although they were not perfectly straight and aligned. Furthermore, it is difficult to perform manually measurements perfectly parallel or perpendicular to the chosen reference and for some objects it may be necessary to sum more measurements or consider intermediate references, thus introducing several errors. For this aspect, one of the main advantages of the reconstructed model is that it allows to easily measure all relative and absolute positions of the points. Regarding the measurements of objects dimensions, all have been made on the final point cloud with CloudCompare or on 2D models with AutoCAD. Errors have been computed as the difference between the manual value and the model one. The overall RMS value is equal to $12 \mathrm{~mm}$; if the measurements made on the part coming from the photogrammetry and on the part coming from the laser scans are considered separately, the resulting RMSE values are $16 \mathrm{~mm}$ and $5 \mathrm{~mm}$ respectively. Table5 summaryze the stats. In terms of objects positions, as done before, all the measurements have been made on 2D models with AutoCAD and errors have been computed as the difference between the manual value and the model one. From the analysis of the sole manual measurements it is possible to deduce that an error in the order of approximately $2-5 \mathrm{~cm}$ was made when taking them, as more measurements made by different references of the same point are not compatible with each other; thus, they cannot be considered as reference values and the resulting RMSE,

\begin{tabular}{|l|c|c|c|c|}
\hline model source & $\#$ & $\begin{array}{c}\text { Mean } \\
{[\mathrm{mm}]}\end{array}$ & $\begin{array}{c}\text { Sigma } \\
{[\mathrm{mm}]}\end{array}$ & $\begin{array}{c}\text { RMS } \\
{[\mathrm{mm}]}\end{array}$ \\
\hline UAV+scan & 38 & -1 & 12 & 12 \\
UAV & 20 & -2 & 16 & 16 \\
scan & 18 & 0 & 5 & 5 \\
\hline
\end{tabular}

Table 5. Statistics of the difference between the measurements of objects dimensions taken manually and taken on the model.

in this case even more than in the previous one, is not indicative of the accuracy of the reconstructed model. All measurements, both manually and on the reconstructed model, have been taken perpendicular to the walls and ends of the tunnel at ground level. Taking such measurements on the 3D point cloud presents similar problems to taking them by hand, as it is difficult to select the points so that the measurements are perfectly perpendicular or parallel to the chosen reference; instead, using 2D models with CAD software it is very easy to trace straight perpendicular lines correctly. Moreover, taking some measurements of this type on the point cloud may be impossible, as the points on the ground or on the wall to be used as a reference may not be present as covered or shielded by other objects; this is not a problem on a $2 \mathrm{D}$ model, as these references projected on a plane appear as lines. In order to extract 2D sections perfectly level, it has been necessary to rotate the point cloud using CloudCompare (as the road surface is inclined) fitting a plane to the points that make up the road and rotate the entire reconstruction by applying the transformation matrix associated to that plane. The resulting RMS value is equal to $38 \mathrm{~mm}$, with all the measurements taken on the laser cloud versus the $5 \mathrm{~mm}$ RMS obtained for the laser cloud in the previous case. It is reasonable to deduce that this difference is due to a worse in manual measurements (as the model is the same). The ability and accuracy in making measurements on the point cloud is not affected only by the accuracy of the reconstruction, but also by the resolution of the cloud. In fact, even if each point is determined with a certain accuracy, the higher or lower point density allows to select with more or less precision the ends of the features to be measured. The more the cloud is dense, the more it is possible to pick a desired point precisely. While the density of the laser point cloud can be simply set during the survey by adjusting the scan resolution, the density of the photogrammetric point cloud is determined mainly by the camera resolution, flight height and data processing settings. When making measurements on the point cloud, some critical situations have been found. For instance, some details have been difficult to recognize if in shaded areas or hidden by overhanging objects or positioned in recesses/cavities of the photogrammetric point cloud. Furthermore, features not fully acquired by laser scans due to obstructions or in correspondence of critical materials cannot be measured. Some of these problems have been solved using 2D models extracted from the point cloud and managed with CAD software, even overcoming the criticality of the resolution as it is not necessary to pick points but sections that can be managed as 2D drawings.

\section{CONCLUSIONS}

This work aimed at evaluating the feasibility of using modern surveying techniques to carry out surveys in the industrial field for the purpose of forensic engineering. This objective has been pursued through an experimental test where a staged car accident has been surveyed with different technologies such UAV photogrammetry and laser scanning. The survey has been compared with the results of a traditional survey made with laser meter ranges and 
tapes. Three different aspects have been analyzed: the practical use of the instruments, the data processing and the usability of the results for forensic purposes. The main advantages of UAV photogrammetry have been the low costs, the flexibility of use, the completeness of the obtained model and the very short acquisition and processing time. Results have been less accurate, mostly because the accuracy of the final model depends, among the other things, on the accuracy of the GPS survey used to scale and georeference the data. It is reasonable expecting few centimetres if satellite positioning is made in RTK mode. About the laser scanning, the main advantages have been the high quality of data, the high accuracy, the high reliability and the fact that data are directly acquired by an active sensor (and therefore a specific error is associated in a univocal and quantifiable manner to every measure). On the other hands, the equipment is very expensive and regarding the survey experience the instrument setup have not been very flexible while the scanning phase showed to be quite timeconsuming, especially over large areas. One factor that is particularly important in the forensic field is the ability to make timely surveys and in a short time. In this respect, UAV photogrammetry proved to be particularly advantageous, especially in large areas with different objects distributed in a scattered manner, where the use of photogrammetry from drone saves hours or days compared to manual measurements or multiple laser scans. Problems would occur in indoor surveys and the integration of both the techniques would overcome this limit. Furthermore, it is also possible to integrate data of exactly the same area collected with different instruments, obtaining redundant information, with the aim of minimizing the measurement uncertainty and generating a model at variable resolution. Another possible scenario could be combining the two technologies to perform a photogrammetric flight and obtain an overall view of an area, detailed with some laser scans at selected points.Overall, the survey experience can be considered successful and the technologies used have proved to be suitable for this purpose. However, some critical issues and possible improvements have to be highlighted. For instance, some materials (in the experiment, windows, bodywork of cars and plates) have not been correctly scanned due to their reflectivity (too low or too high) or the GSD obtained in the chosen photogrammetric project is $3.97 \mathrm{~cm}$, resulting in absolute at centimetre level that could be considered too high. However, these accuracies can be improved using cameras of better quality, obtaining GSD less than one $\mathrm{cm}$ at the same flight height. Summarizing, when compared with the traditional methods for surveying accident site, the geomatics approach proved to furnish fast and timely surveys, acceptable/high accuracy, simultaneous acquisition of many points, the three dimensional digital reconstruction that can be managed and processed at any time, permanent preservation of data and reconstructed scene so that measurements can be repeated several times, georeferenced data, time stamp (date and hour) of the GPS readings, no need of entering into physical contact with the objects and no need of physically entering the area to be surveyed.

\section{REFERENCES}

Ardestani, S. M., Jin, P. J., Volkmann, O., Gong, J., Zhou, Z. and Feeley, C., 2016. 3D Accident Site Reconstruction Using Unmanned Aerial Vehicles (UAV). Technical report, Transportation Research Board 95th Annual Meeting, Washington DC, United States.

Brach, R. and Brach, M., 2011. Vehicle accident analysis and reconstruction methods, second edition. SAE International.
Buck, U., Naether, S., Braun, M., Bolliger, S., Friederich, H., Jackowski, C., Aghayev, E., Christe, A., Vock, P., Dirnhofer, R. and Thali, M., 2007. Application of 3D documentation and geometric reconstruction methods in traffic accident analysis: with high resolution surface scanning, radiological MSCT/MRI scanning and real data based animation. Forensic science international 170 , pp. $20-8$.

Carter, N., Hashemian, A., Rose, N. A. and Neale, W. T., 2016. Evaluation of the Accuracy of Image Based Scanning as a Basis for Photogrammetric Reconstruction of Physical Evidence. SAE Technical Paper.

Colwill, S., 2016. Low-cost crime scene mapping: reviewing emerging freeware, low-cost methods of 3D mapping and applying them to crime scene investigation and forensic evidence. Technical report, Murdoch University, Australia.

Du, X., Xianlong, J., Zhang, X., Shen, J. and Hou, X., 2009. Geometry features measurement of traffic accident for reconstruction based on close-range photogrammetry. Advances in Engineering Software 40, pp. 497-505.

Eyre, M., Foster, P., Speake, G. and Coggan, J., 2017. Integration of laser scanning and three-dimensional models in the legal process following an industrial accident. Safety and health at work 8(3), pp. 306-314.

Fagandini, R., Federici, B., Ferrando, I., Gagliolo, S., Pagliari, D., Passoni, D., Pinto, L., Rossi, L. and Sguerso, D., 2017. Evaluation of the laser response of leica nova multistation ms60 for 3d modelling and structural monitoring. pp. 93-104.

Fowle, K. and Schofield, D., 2011. Visualising forensic data: Investigation to court. In: Proceedings of the 9th Australian Digital Forensics Conference, Perth, Australia.

Fraser, C. S., Hanley, H. B. and Cronk, S., 2005. Close-range photogrammetry for accident reconstruction. Optical $3 \mathrm{D} \mathrm{Mea-}$ surements VII 2, pp. 115-123.

Fricke, L., 1990. Traffic accident reconstruction: Vol.2. Technical report, Traffic Institute, Northwestern University, Evanston, IL, USA.

Pagliari, D. and Pinto, L., 2018. Use of fisheye parrot bebop 2 images for $3 \mathrm{~d}$ modelling using commercial photogrammetric software. ISPRS - International Archives of the Photogrammetry, Remote Sensing and Spatial Information Sciences XLII-2, pp. 813-820.

Pagounis, V., Tsakiri, M., Palaskas, S., Biza, B. and Zaloumi, E., 2006. 3d laser scanning for road safety and accident reconstruction. In: Proceedings of the XXIIIth international FIG congress, Hong Kong, China, pp. 8-13.

Pu, S., Rutzinger, M., Vosselman, G. and Elberink, S., 2011. Recognizing basic structures from mobile laser scanning data for road inventory studies. ISPRS Journal of Photogrammetry and Remote Sensing 66(6), pp. 28-39.

Randles, B., Jones, B., Welcher, J., Szabo, T., Elliott, D. and MacAdams, C., 2010. The accuracy of photogrammetry vs. hands-on measurement techniques used in accident reconstruction. SAE Technical Paper.

Smith, M. L., 2015. Regulating law enforcement's use of drones: The need for state legislation. Harv. J. on Legis. 52, pp. 423.

Su, S., Liu, W., Li, K., Yang, G., Feng, C., Ming, J., Liu, G., Liu, S. and Yin, Z., 2016. Developing an unmanned aerial vehiclebased rapid mapping system for traffic accident investigation. Australian journal of forensic sciences 48(4), pp. 454-468. 